\title{
Research on the Impact of Social Circles on Self-Brand Connection: Regulation of Self-Awareness and Brand Value*
}

\author{
Shenghong Ye, Jia Li, Zhaobin Zeng, Shuang Hao \\ Management School, Jinan University, Guangzhou, China \\ Email: 787477797@qq.com
}

Received 15 February 2015; accepted 7 April 2015; published 9 April 2015

Copyright (C) 2015 by authors and Scientific Research Publishing Inc.

This work is licensed under the Creative Commons Attribution International License (CC BY). http://creativecommons.org/licenses/by/4.0/

(c) (i) D Den Access

\begin{abstract}
In this article we categorize social circles by sense of belonging and explore the relation between social circle types and self-brand connection (SBC). Furthermore, we research the mechanism of how the impact of social circles on consumers' SBC is influenced by self-awareness within a particular social circle and by brand value. Our findings show that 1) the sense of belonging toward social circles has positive influence on SBC; 2 ) consumers' self-awareness moderates the impact of social circles on SBC; 3 ) the type of brand value moderates the impact of social circles on SBC. Finally, several suggestions are derived for local management practice in China.
\end{abstract}

\section{Keywords}

Social Circles, Self-Brand Connection, Public Self Consciousness, Sense of Belonging, Brand Value

\section{Introduction}

Companies have always been pursing close connection between their brands and consumers, which falls into the field of self-brand connection (SBC). By definition, SBC refers to consumers' construction, reinforcement and expression of their selves through a particular brand. Escalas and Bettman discovered that Consumers' loyalty toward a brand increases as self-brand connection goes up, which then helps boost brand equity [1] [2]. It has become a big focus among both scholars and company managers how SBC can be elevated to preferable levels.

Most researches on SBC are embedded in western communities and Chinese researchers tend to draw on the

"Ministry of National Education Fund Project "Self-Brand Relationship Links under Chinese Cultural Background of: Research of Differential Pattern Based on the Perspective” (12YJC630269); Innovative Projects of Jinan University Enterprise Institute, “Consumers-Brand Relationship under Chinese Cultural Background: Based on the Perspective of Social Circle” (2013CP006).

How to cite this paper: Ye, S.H., Li, J., Zeng, Z.B. and Hao, S. (2015) Research on the Impact of Social Circles on Self-Brand Connection: Regulation of Self-Awareness and Brand Value. Open Journal of Business and Management, 3, $155-162$. http://dx.doi.org/10.4236/ojbm.2015.32015 
overseas findings. However, it is in fact a strong social behavior that consumers build self-image, sense of belonging and social recognition through purchases [3]. Western communities are based on individuality, which is a far cry from Confucian culture in the east. Particularly, in Chinese society where traditional customs and group awareness have been being highly valued for millenniums, consumers' perceptions of a symbolic brand are even more influenced by association differentiation structure, social circles and values. As a result, we use Chinese consumers' self-awareness and brand value as moderator variables and explore the collaborative effects of consumers' social circles and their sense of belonging on SBC.

\section{Conceptualization and Theoretical Framework}

Self-brand connection describes to what degree consumers associate the brand and self-concept. Chaplin and John believed that consumers' SBC involves three factors: brand association, self-concept and self-image comparison [4]. Brand association, especially when it is related to a particular group, plays an essential role in the influence on SBC [5]. Under Chinese cultural circumstance, a typical group which forms people's social circle is constantly influenced by Confucian culture and this leads to a fact that Chinese people are no individuals but active participants in families, neighborhoods, nations and other existing groups across the world. Fei HsiaoTung made a famous argument that Chinese's interpersonal relationship is not a bundle of sticks but in fact the ripples on a water surface caused by a stone and everyone serves as the center of each circle [6]. Consumers' social circles may change when they go with different people, so Chinese social circles can impact people's fusion with a brand then further impact consumers' SBC.

Some existing researches focus on reference groups which are apparently different from social circles [7]. Reference groups have to do with group impact and mostly refer to the groups which people do not belong to, such as desirable groups and distasteful groups. By contrast, social circles emphasize that everyone serves as the center of his or her own circle, which involves ethics-centeredness, association differentiation and benefits. In a Chinese social circle, each member pays a great deal of attention to others' suggestions because one is unlikely to fit in well unless he or she follows the ideas adopted by the circle. As a matter of fact, searching for sense of belonging is a major way of behavior for Chinese people. Therefore, we believe it is necessary to look into the effects of social circles with different levels of belonging sense on consumers' SBC.

Another factor which can affect SBC is self-concept which refers to individuals' reflection of psychological process and content. Brands can form some psychological meanings both on individual level and social level. Different self-concepts drive different consumption motivation and a unique self leads to the occupation and interpretation of a brand. Self-concept consists of private self-consciousness and public self consciousness. In the case of Chinese social circles, if consumers focus more on the visible content which others can comment, they display the public self consciousness. We attempt to find out social circles' influence on SBC based on self-awareness. In addition, because brand value can help to mold a brand's symbolic meaning within a circle, in the final section we explore the mechanism of how brand value impacts the link between social circles and SBC. Figure 1 provides a schematic representation of the framework.

\section{Hypoheses}

\subsection{Social Circles' Impact on Self-Brand Connection}

Social circles have significant influence on their members in terms of attitudes, willingness, behaviors and pref-

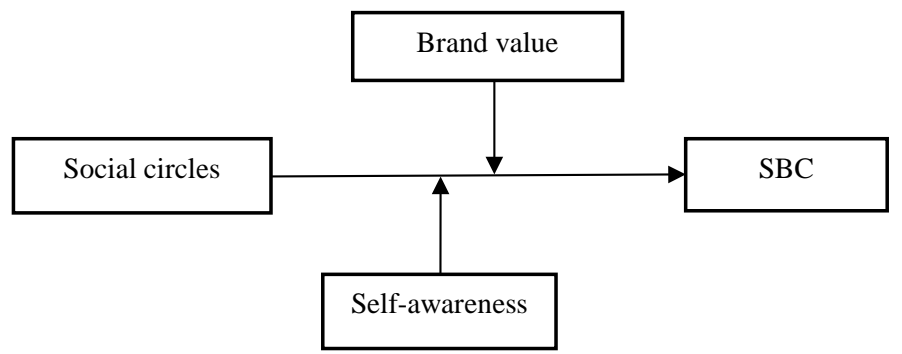

Figure 1. A framework for the link between social circles and SBC. 
erence and in turn members attempt to build their self-image [8], friend affiliation and social recognition [9] [10] within the circles through consumption behaviors. Social circles are what we turn to for reference and are important sources of brand image and brand association, so they help with consumers' self-fashioning.

Social circles' impact on value mainly comes from individuals' desire for self-improvement, reinforcement of belonging sense. And friend affiliation and recognition drive consumers to connect with groups within the circle. So consumers tend to have different value tendencies when they are on different levels of belonging sense toward social circles. They are more likely to choose the brand most used in the social circle with higher belonging sense and to establish connection with it. Under Chinese cultural circumstance, social circles are social networks where people adopt different criteria of recognition from those in western communities. Li Youmei adopted a class identity-centered approach and described social identity as combination of common beliefs, values and behavioral tendencies which actually is some kind of collective spirit [11]. Compared to benefit-based social identity, belonging sense based social identity are more stable. Social circles can be divided into high sense belonging circles and low sense belonging circles. As a result, the brand chosen by a social circle have different impact on Chinese consumers than on western consumers and the connection with the brand will become stronger as circle members' sense of belonging increases. That is because stronger connection with the brand means affirmation of the social circle. We propose the first hypothesis as follows:

H1: Consumers have higher SBC in high belonging sense social circles than in low belonging sense social circles.

\subsection{Regulation of Self-Awareness}

Self is the perception and evaluation of oneself on many aspects including what kind of person one believes he/ she will become, what kind of person one dreams of becoming and fears becoming. This self perception can influence people's behaviors and in turn people can express themselves through particular behaviors. In a social circle, the level of consumers' public self consciousness affects their connection with a brand. Some researchers discovered that consumers' self-awareness plays a vital role in SBC within a group. Fenigstein, Seheier and Buss divided self-awareness into two groups: secret aspects of self that others can by no means know, and visible aspects of self that are closely related to others. They defined the former group as private self and the latter as public self and argued that private self and public self can exist together in one person. People who have higher self-awareness are considered to be public self conscious and those with lower self-awareness to be privateself conscious.

Chinese culture places great importance on harmony so that self-awareness which is closely related to others performs a key function on consumers' brand connection within social circles. The public self conscious consumers - the ones with higher self-awareness - tend to pay more attention to how other members think of them. Regardless of the level of belonging sense, their connections with the circle's brand increases. That is to say, this type of consumers tends to love what others love. In the case of private self conscious consumers-the ones with lower self-awareness, they are more aware of group norms in high belonging sense circles and are more likely to be influenced by other circle member. Therefore, consumers' public self awareness moderates SBC and SBC in high belonging sense circles is higher than in low belonging sense circles. We then propose the hypotheses as follows:

H2: Self-awareness moderates the social circles' impact on SBC.

H2a: For the public self conscious consumers, there is no significant difference in the impact of social circles with different levels of belonging sense on SBC.

H2b: For the private self conscious consumers, there is higher SBC in high belonging sense social circles than in low belonging sense social circles.

\subsection{Regulation of Brand Value}

The third factor which affects SBC is consumers' self-image comparison. The match between a brand and a consumer's image has been a big focus for researchers since Fournier proposed personification of brands [12]. Within a social circle, whether or not brand value matches a consumer's image play a key role in the consumer's connection with the brand. Brand value consists of functional value and symbolic value. Researchers believe that not only do the consumers care about the products' functional characteristics but about the satisfaction of their psychological needs, such as conformity, uniqueness and reputation as well as self-improvement, role posi- 
tioning and social group identity [13]. Based on that, inspired by studies on sociology and social psychology fronts, researchers begin to link a brand' symbolic meaning with the construction and development of consumers' self-concept.

Consumers are mostly attracted by the products or brands comparable to their social status [14]. The brands with prominent function values do not have close emotional link with consumers because they have unapparent symbolic meaning in social circles. In high belonging sense circles, consumers are more interested in connection with the circles, while in low belonging sense circles they focus more on their interest. As a result, consumers' SBC in high belonging sense circles is weaker than in low belonging sense circles. When it comes to the brands with prominent symbolic values, brands better symbolize social circles. Accordingly, consumers who have better sense of belonging tend to have stronger SBC. Hypotheses are proposed as follows:

H3: Brand value moderates social circles' impact on SBC.

H3a: For brands with prominent functional values, consumers have weaker SBC in high belonging sense social circles than in low belonging sense circles.

H3b: For brands with prominent symbolic values, consumers have stronger SBC in high belonging sense social circles than in low belonging sense circles.

\section{The Empirical Approach and Findings}

\section{Preliminary Test}

The purpose of preliminary test is to determine the product types and brands for the master test. The requirements for products and brands are that 1) products are basically used by everyone, 2) brands enjoy favorable recognition rates for the products, 3 ) brands have both functional values and symbolic values, that is to say, some consumers choose them for their functional values and others choose them for symbolic values.

In order to determine products and brands, we carried out a small scale survey where pedestrians around campus were asked questions. The survey respondents were randomly selected and there were a total of 16 respondents for this preliminary test. They were required to choose brands that they often used from 5 common product types including watches, garments, handbags, personal computers and skin care. Then respondents were told to describe the brand-related events that had happened in their social circles. Results show that watches bring about the most significant association differentiation (87\% response rate), handbags rank second (79\% response rate), the rest of products have less significant effects. So handbags are selected as an object of this study. During the second questionnaire preliminary test, we chose 10 handbag brands and targeted college students' social circles with the purpose to distinguish high belonging sense social circles from low belonging sense circles and to measure the sense of belonging and familiarity brought by each brand (7 Likert scale). There are 52 college students as participants for this preliminary test (27 females and 25 males). Eventually, we choose Chanel as the brand for low belonging sense circles and Nike as the brand for high belonging sense circles. The sense of belonging from Chanel $(\mathrm{M}$ Chanel $=2.2)$ is lower than that from Nike $(\mathrm{M}$ Nike $=5.93)(\mathrm{p}<0.001)$ and there is no significant difference in familiarity with these two brands ( $p>0.05)$.

\section{Study 1: Social circles' impact on SBC and regulation of self-awareness}

1. Study design and process

This study adopts a $2 \times 2$ approach which mixes social circles types-high belonging sense circles and low belonging sense circles with self-awareness-public self consciousness and private self consciousness. Among all these factors, Social circles types serve as in-group factors. At first, subjects are briefed on Nike and Chanel products then on the meaning of real-life social circles. Next they are told to recall two types of social circles that they belong to-a high belonging sense circle and a low belonging sense circle. Then through situation simulation subjects are to consider the circumstance that they need to buy a suitable handbag for job or personal reasons within these two circles respectively, by which, the level of SBC with different circles can be measured. Finally, we measure consumers' sense of belonging and self-awareness.

2. Scale design

Measurement of variables in this study mainly draws on the well-tried Likert7 scales where 1 represents strongly disagree and 7 represents strongly agree. Measurement of sense of belonging as well as SBC relies on the scales adopted by Escalas and Bettman [1] [2] where there are 3 indicators for sense of belonging and 7 indicators for SBC. Self-awareness is measured by the scale used by Fenigstein, Seheier and Buss which has 7 indicators. 


\section{Manipulation test}

Study 1 uses online questionnaires and 202 out of 210 questionnaires are collected with the $96.2 \%$ effectiveness rate. 93 males and 109 females are involved with the age band of 20 to 30 . All the main variables in study 1 have satisfactory reliability with Cronbach's $\alpha$ value over 0.9 . Variable manipulation shows that (1) in terms of brand familiarity test, subjects have favorable familiarity with both brands(Nike $M=6.62$, Chanel $M=6.21$ ) without significant difference $(F(2,88)=0.213$, p > 0.05), (2) in terms of sense of belonging toward social circles, for high belonging sense circles ( $\mathrm{M}$ high $=6.04$ ), and low belonging sense circles $(\mathrm{M}$ low $=3.22$ ), social circle types are successfully manipulated $(\mathrm{p}<0.001)$. Results show that variable manipulation is successful.

4. Hypothesis test

By means of SPSS16.0, variance analysis of the main effect-social circles' impact on SBC shows that the value of SBC in high belonging sense circles ( $\mathrm{M}$ high) is 5.45 while the value in low belonging sense circles( $\mathrm{M}$ low) is 3.96. SBC is significantly different between high and low belonging sense circles $(F(1,201)=105.47, p$ $<0.001$ ), so hypothesis 1 is proven.

Self-awareness is divided into public self consciousness and private self consciousness through the median rating approach. Then $2 \times 2$ analysis of variance finds that social circles have significant interaction with self-awareness $(\mathrm{p}<0.001)$. Findings are showed as follows Table 1.

Further analysis discovers that for the pubic self conscious consumers, there is no significant difference in SBC between high belonging sense circles and low belonging sense circles ( $p>0.05)$ (H2a proven). On the other hand, for private self conscious consumers, SBC in high belonging sense circles is stronger than in low belonging sense circles ( $<0.001$ ) (H2b proven). The finding is displayed in Figure 2.

\section{Study 2: Social circles' impact on SBC and regulation of brand value}

1. Study design and process

This study adopts a $2 \times 2$ approach which mixes social circles types-high belonging sense circles and low belonging sense circles with brand value-functional value and symbolic value. Study process is the same as study 1 .

2. Manipulation test

Both paper questionnaires and electronic questionnaires are distributed and 202 out of 215 questionnaires are collected with the effectiveness rate of $93.9 \%$. There are 100 males and 102 females involved with the age band of 20 to 30. All the main variables in study 1 have satisfactory reliability with Cronbach's $\alpha$ value over 0.9. Both

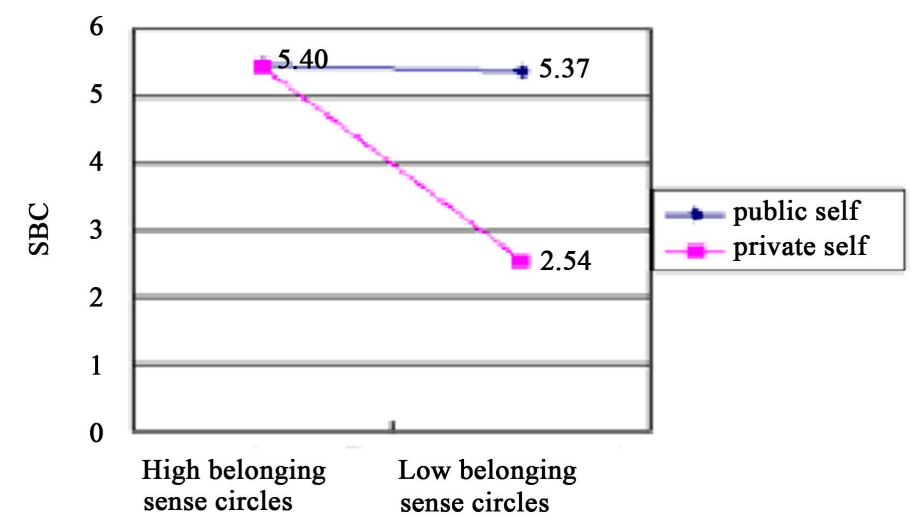

Figure 2. Impact of social circles and self-awareness on SBC.

Table 1. Impact of social circles and self-awareness on SBC.

\begin{tabular}{cccc}
\hline & Variables & SBC & SD \\
\hline Pubic self consciousness & High belonging sense circles & 5.46 & 0.248 \\
& Low belonging sense circles & 5.37 & 0.266 \\
Private self consciousness & High belonging sense circles & 5.43 & 0.221 \\
& Low belonging sense circles & 2.54 & 0.248 \\
\hline
\end{tabular}

Data source: research data. 
brand familiarity and sense of belonging are successfully manipulated. In terms of the test of brands' value attributes, results show that subjects have significant difference in value perception of these two brands( $\mathrm{M}$ Nike $=$ 3.31, M Chanel $=5.33, \mathrm{t}(90)=-14.541, \mathrm{p}<0.01)$. Results show that manipulation in study 2 is successful.

3. Hypothesis test

Variance analysis of $2 \times 2$ (high belonging sense circles and low belonging sense circles mixed withfunctional value and symbolic value) with SBC shows that social circles have significant interaction with brand value ( $<<$ 0.001). The findings are displayed in Table 2.

Specifically, for brands with prominent functional value, SBC in high belonging sense circles is weaker than in low belonging sense circles ( $<0.001$ ) (H3a is proven). On the other hand, for brands with prominent symbolic value, SBC in high belonging sense circles is stronger than in low belonging sense circles ( $p<0.001)$ (H3b is proven). The results are displayed in Figure 3.

\section{Conclusions and Managerial Implitions}

Social circles have great impact on Chinese people's daily life including perception and selection of a brand. This article explores what effects social circles on different levels of belonging sense can put on consumers' SBC under Chinese cultural circumstance. In addition, based on the influencing factors of SBC, self-awareness and brand value are brought in as moderator variables and their relation is examined. The findings show that 1) the sense of belonging toward social circles positively influences SBC; 2) consumers' self-awareness-public and private self consciousness-moderates the impact of social circles on SBC. When public self consciousness outweighs private self consciousness, consumers' SBC is insignificantly influenced by the level of belonging sense toward social circles. When private self consciousness dominates, consumers' SBC in high belonging sense circles is stronger than that in low belonging sense circles; 3) the types of brand value-functional value and symbolic value-moderate the impact of social circles on SBC. If a brand is more of functional value, consumers' SBC in high belonging sense circles is weaker than that in low belonging sense circles. When it comes

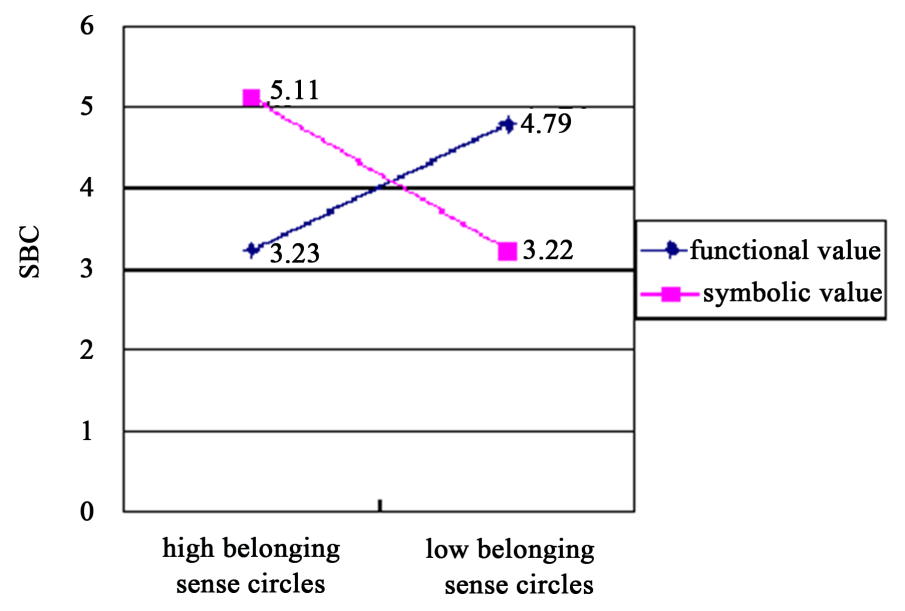

Figure 3. Impact of social circles and brand value on SBC.

Table 2. Impact of social circles and brand value on SBC.

\begin{tabular}{lccc}
\hline & Variables & SBC & SD \\
\hline Functional value & High belonging sense circles & 3.23 & 0.345 \\
& Low belonging sense circles & 4.79 & 0.443 \\
Symbolic value & High belonging sense circles & 5.11 & 0.491 \\
& Low belonging sense circles & 3.22 & 0.448 \\
\hline
\end{tabular}

Data source: research data. 
to a brand which is focused more on symbolic value, consumers' SBC in high belonging sense circles is stronger than that in low belonging sense circles.

Several managerial implications are derived from the findings of this study.

1. Managers should place more importance on consumer culture within social circles. China is a major consumption powerhouse where social circles play essential roles in consumption, which is tested and proven in this study. Companies are supposed to take advantage of social circles and then come up with relevant brand promotion and product positioning strategies. Orion egg pie serves a good example in utilizing the effects of social circles. Orion's advertisements and other kinds of promotion stick to the ideas that we are good friends and that we go with good friend our entire life. After fully understanding the core elements that make good friends, Orion amplifies them and turns target customers' recognition of their good friend to the recognition of the brand and the product.

2. Various circles cultures should be promoted depending on the types of consumers' self-awareness. The impact of social circles on SBC can be significantly moderatedby consumers' self-awareness, which implies that companies should attempt to embrace all kinds of views towards social circles as long as it will not affect other circle members. It is advisable that advertisements convey dual ideas of which one is aimed at public self conscious consumers and the other targets private self conscious consumers. For instance, in Japan there were once a series of Fanta advertisements which display consumers' various lifestyles through diverse situations and sum up with the idea that drinking Fanta can help get rid of unhappiness. These advertisements are so inclusive that they involve various types of consumers' recognition of the brand and derive the values of emotional satisfaction related to the brand.

3. Diverse circle cultures are supposed to be created according to brand value. Within social circles, consumers' SBC varies depending on the value types of brands. Companies need to have a good knowledge of their brand value and use the value as a foundation for creating circles cultures for their own brands. If the brand is focused on practical value such as washing powder and detergent, then social circles play a less important role in promotion. However, when it comes to brands which are more of symbolic value such as watches and cars, basing the promotion strategies on the sense of belonging can better impress Chinese consumers who value social circles and establish preferable connections between consumers and brands.

\section{References}

[1] Escalas, J.E. and Bettman, J.R. (2003) You Are What They Eat The Influence of Reference Groups on Consumer Connections to Brands. Journal of Consumer Psychology, 13, 339-348. http://dx.doi.org/10.1207/S15327663JCP1303_14

[2] Escalas, J.E. and Bettman, J.R. (2005) Self-Construal Reference Groups and Brand Meaning. Journal of Consumer Research, 32, 378-389.

[3] Dreze, X. and Nunes, J. (2009) Feeling Superior: The Impact of Loyalty Program Structure on Consumer Perception of Status. Journal of Consumer Research, 35, 890-905. http://dx.doi.org/10.1086/593946

[4] Chaplin, L.N. and John, D.R. (2005) The Development of Self-Brand Connections in Children and Adolescents. Journal of Consumer Research, 32, 119-129. http://dx.doi.org/10.1086/426622

[5] Muniz Jr., A.M. and O’Guinn, T.C. (2001) Brand Community. Journal of Consumer Research, 27, 412-432. http://dx.doi.org/10.1086/319618

[6] Fei, X.T. (1998) Rural China and Fertility Regimes. Peking University Press, Beijing.

[7] Du, W.Q., Yu, C.L. and Zhao, P. (2009) Type of Reference Group and Self-Brand Connection. Acta Psychologica Sinica, 2, 156-166.

[8] Chernev, A., Hamilton, R. and Gal, D. (2011) Competing for Consumer Identity: Limits to Self-Expression and the Perils of Lifestyle Branding. Journal of Marketing, 75, 66-82. http://dx.doi.org/10.1509/jmkg.75.3.66

[9] Barwise, T.P. (1992) Brand Equity: Snark or Boojum? International Journal of Research Marketing, 10, 93-104.

[10] Chattaraman, V., Rudd Nancy, A. and Lennon Sharron, J. (2010) The Malleable Bicultural Consumer: Effects of Cultural Contexts on Aesthetic Judgments. Journal of Consumer Behaviour, 9, 18-31.

[11] Li, Y.M., Xiao, Y. and Huang, X.C. (2007) Social Identity. A Structural View Analysis—As an Example of the United States, Germany and Japan. Shanghai People’s Publishing House, Shanghai.

[12] Fournier, S. (1998) Consumers and Their Brands: Developing Relationship Theory in Consumer Research. Journal of Consumer Research, 24, 343-373. http://dx.doi.org/10.1086/209515 
[13] Park, C.W. and Lessig, V.P. (1977) Students and Housewives: Difference in Susceptibility to Reference Group Influence. Journal of Consumer Research, 3, 102-110.

[14] Forehand, M.R., Deshpandé, R. and Reed II, A. (2002) Identity Salience and the Influence of Activation the Social Self-Schema on Advertising Response. Journal of Applied Psychology, 87, 1086-1099. 